\title{
Criminologie
}

\section{Les droits de la jeunesse et le projet de loi C-192}

\section{Alice Parizeau}

Volume 8, numéro 1-2, 1975

Délinquance juvénile au Québec

URI : https://id.erudit.org/iderudit/017037ar

DOI : https://doi.org/10.7202/017037ar

Aller au sommaire du numéro

Éditeur(s)

Les Presses de l'Université de Montréal

ISSN

0316-0041 (imprimé)

1492-1367 (numérique)

Découvrir la revue

Citer cet article

Parizeau, A. (1975). Les droits de la jeunesse et le projet de loi C-192.

Criminologie, 8(1-2), 29-52. https://doi.org/10.7202/017037ar d'utilisation que vous pouvez consulter en ligne.

https://apropos.erudit.org/fr/usagers/politique-dutilisation/ 
LES DROITS DE LA JEUNESSE

ET LE PROJET DE LOI C-192

Alice Parizeau 
Il n'est certainement pas original d'affirmer que la société canadienne a beaucoup évolué au cours de la dernière décennie. Il est fort intéressant cependant d'essayer de mesurer cette évolution en termes de changements législatifs.

À cet égard, le Bill Omnibus de 1969 demeure on ne peut plus significatif. En effet, malgré l'opposition du puritanisme protestant et de l'approche moralisatrice, issue d'une certaine tradition catholique, le ministre de la justice de l'époque est parvenu à le présenter en Chambre et à le faire voter. Déjà, on peut voir là la preuve d'une évolution de la population canadienne en ce qui a trait à l'échelle des valeurs qu'elle est prête à défendre. En ce qui concerne la réaction à l'égard de la criminalité, on a l'impression d'assister à un accroissement du degré de permissivité ou à un recul de l'approche punitive, les deux formes de réactions, bien que difficilement mesurables, ayant des conséquences comparables.

D'une manière générale, on peut prétendre donc que le bilan des cinq dernières années, en termes de réformes législatives, se solde par la décriminalisation de certains délits, dont l'homosexualité entre adultes consentants, l'abandon de la théorie du crime et du châtiment qu'illustre le fait de suspension de la peine capitale, et une progression vers l'optique de resocialisation, à l'opposé de celle de punition, qui se traduit dans des législations concernant notamment la libération sur parole, ou encore une plus large utilisation de la mesure de probation.

Or, phénomène curieux, tout se passe comme si l'ensemble des politiques nouvelles ne devait toucher à la justice des mineurs. 
Comme si la société des adultes refusait d'assumer des risques, voire même remplir ses devoirs élémentaires à l'égard de la protection de leurs droits.

\section{A. LA LOI DES JEUNES DÉLINQUANTS}

Certes, on a accepté de reconnaître que la Loi de jeunes délinquants, qui date de 1929 et qui de toute façon n'est qu'une copie fort mal adaptée au contexte canadien, déjà à cette époque de la législation d'un état américain, n'est plus satisfaisante, il n'en reste pas moins qu'elle est toujours en vigueur. C'est, en effet, une loi qui reflète des attitudes répressives tout à fait inacceptables d'une société mal réveillée de son passé rural, qui s'industrialise et qui veut contrôler avec une sévérité totale les comportements de cette main-d'œuvre bon marché qui est représentée, au Canada comme en Grande-Bretagne d'ailleurs, par les mineurs.

C'est ainsi qu'on peut lire dans le texte de la loi : «Nul ne doit être déclaré coupable d'une infraction... lorsqu'il est âgé de moins de sept ans », ce qui est on ne peut plus édifiant, compte tenu du fait que le concept même de la culpabilité, tel que perçu par un enfant de sept à douze ans, dépend en réalité du type d'éducation qu'il reçoit.

En ce qui a trait à la définition de la délinquance, à l'article I, (par. 2) on apprend que : «Jeune délinquant » signifie un enfant qui commet une infraction à quelqu'une des dispositions du Code criminel, ou d'un statut fédéral ou provincial, ou d'un règlement ou ordonnance d'une municipalité, ou qui est coupable d'immoralité sexuelle, ou de toute forme semblable de vice, ou qui, en raison de toute autre infraction, est passible de détention dans une école industrielle ou maison de correction pour les jeunes délinquants, en vertu des dispositions d'un statut fédéral ou provincial. 》

Autant dire que la société s'arroge le droit absolu d'évaluer la nocivité des comportements d'un mineur et que bien que les adultes consentants ont désormais droit de pratiquer l'homosexualité comme bon leur semble, le mineur, lui, s'il embrasse une fille sur un banc public, peut être condamné comme «coupable d'immoralité sexuelle $\$$.

On peut objecter à cela que la loi est appliquée par des juges responsables et capables d'un discernement suffisant pour 
rendre justice. Cela est incontestablement vrai, mais il n'en reste pas moins que certains textes législatifs reflètent la philosophie qui prévaut dans la société et que tel est le cas en ce qui a trait aux jeunes. En confirmant les réactions punitives et la conception de l'autorité absolue, dont a fait preuve la société canadienne, la Loi des jeunes délinquants reconnaît le statut quo. Or, par le passé, cela a donné lieu à des abus désormais tout simplement incroyables.

A cet égard il est intéressant encore aujourd'hui de citer le rapport de la Commission Royale d'Enquête sur le Capital et le Travail qui a siégé au début du siècle. Lors des audiences tenues à Montréal, les Commissaires ont entendu, entre autres, le témoignage suivant :

Q. : Vous êtes ouvrier cigarier?

R. : Oui, monsieur.

Q.: Quel âge avez-vous ?

R. : J'ai eu 14 ans le 10 janvier dernier.

Q. : En disant que vous êtes un ouvrier cigarier, vous voulez dire que vous avez servi votre apprentissage, n'est-ce pas ?

R. : Oui, monsieur.

Q. : Combien de temps ?

R. : 3 ans.

Q. : Vous avez commencé à travailler à 11 ans ?

R. : Oui, monsieur...

Q. : Combien travaillez-vous d'heures par jour?

R. : Des fois, dix heures, d'autres fois, huit heures ; c'est selon comme on le voulait...

Q. : Pourquoi avez-vous payé des amendes, vous en rappelezvous ?

R. : Des fois, c'est parce qu'on parlait trop ; plus souvent qu'autrement c'était pour ça.

Q. : Vous n'avez jamais été battu ?

R. : Oui. Pas battu pour dire que cela m'a fait du dommage, mais ils arrivaient à nous autres, et des fois, on s'adonnait à mal tailler notre robe; ils nous donnaient un coup de poing sur la tête.

Q. : Est-ce que c'est la coutume de battre les enfants comme cela?

R. : Souvent.

Q. : Avez-vous été battu pendant la première année de votre apprentissage? 
R. : Oui, monsieur...

Q. : Avez-vous vu battre d'autres enfants?

R. : Oui...

Q. : Avez-vous connaissance d'une fabrique dans laquelle il y a un cachot?

R. : Oui, monsieur.

Q. : Avez-vous vu des enfants mis dans ce cachot?

R. : Oui, monsieur.

Q. : Des enfants de quel âge ?

R. : Je ne puis pas dire l'âge.

Q. : Plus jeunes que vous ?

R. : Non, monsieur.

Q. : Pourquoi ont-ils été mis au cachot?

R. : Parce qu'ils perdaient du temps.

Q. : Qui est-ce qui les mettait au cachot?

R. : C'est l'homme qui gardait la presse.

Q. : Savez-vous si cet homme porte une médaille de constable ?

R. : Oui, monsieur.

Q. : Les enfants criaient-ils ?

R. : Non.

Q. : Ont-ils été menés brutalement au cachot ?

R. : Non, monsieur.

Q. : Combien de temps restaient-ils au cachot, généralement ?

R. : Il en est resté jusqu'à 7 heures.

Q. : Quand est-ce qu'on les avait mis dedans?

R. : Dans l'après-midi.

Q. : Est-ce à 7 heures le soir, ou pendant 7 heures de temps ?

R. : 7 heures du soir. Ils les mettaient dans l'après-midi jusqu'à 7 heures du soir.

Q. : A quelle heure les ouvriers quittent-ils la fabrique ?

R. : Généralement à cinq heures et quelques fois à six.

Q. : Voulez-vous dire que ces enfants sont restés dans le cachot après que les ouvriers avaient quitté la fabrique ?

R. : Oui, monsieur.

Q. : Qui est-ce qui les faisait sortir : est-ce celui qui les avait mis?

R. : Oui, monsieur ; je pense bien; je ne l'ai pas vu.

Q. : Ce cachot est-il chauffé ?

R. : Je ne le sais pas.

Q. : À quel étage de la fabrique ce cachot est-il situé ?

R. : Dans la cave. 
Q. : Y a-t-il une fournaise dans la cave ?

R. : Oui, monsieur.

Q. : Est-ce que le cachot est près de la fournaise ?

R. : Non, monsieur.

Q. : Y a-t-il une fenêtre dans ce cachot ?

R.: Non.

Q. : Quand les enfants étaient là vous n'avez jamais entendu appeler pour qu'on les fît sortir ?

R. : Non, monsieur.

Q. : À quel âge avez-vous quitté l'école?

R. : Dix ans et demi.

Q. : Savez-vous lire et écrire ?

R. : Un peu.

Q. : Savez-vous faire vos quatre règles ?

R. : Je ne sais pas si je m'en souviens.

Q. : Etes-vous orphelin?

R. : Non, monsieur.

Depuis l'époque où les commissaires se demandaient pourquoi les enfants-ouvriers enfermés dans un cachot n'osent pas appeler à l'aide, la société a beaucoup évolué, mais il n'en reste pas moins significatif que la Loi des Jeunes Délinquants, toujours en vigueur, date à peu près de la même période...

Certes il s'agit là d'une des plus sinistres images de notre société, qui fort heureusement, appartient au passé, il n'en reste pas moins que la Loi des Jeunes Délinquants, dans sa forme comme dans son esprit, demeure un texte législatif, qui implicitement accepte ou encore sanctionne les agissements de ce type. Le terme de délinquance juvénile, tel qu'il existait dans la loi jusqu'en 1973, comprenait non seulement des délits, mais aussi des comportements désignés sous les termes de « ingouvernable », " incorrigible 》, ou encore " sexuellement immoral », ce qui renforçait toutes les possibilités d'injustice pouvant être commises et qui ont été souvent commises à l'égard des enfants.

A titre d'exemple, mentionnons que le Canada demeure le seul pays occidental où la responsabilité pénale commence à l'âge de sept ans et où en raison de la contradiction entre l'exemption de responsabilité criminelle prévue par le Code criminel et la Common Law et la Loi sur les jeunes délinquants, même des enfants de moins de sept ans peuvent être jugés en vertu d'une loi criminelle, ce qui nous paraît, sur le plan international, une 
particularité dont le mérite demeure douteux, pour ne pas dire plus.

En d'autres termes, la loi ne protège d'aucune façon le droit de l'enfance en ce qui a trait au concept de culpabilité et à la définition de la délinquance. Ajoutons que la situation demeure tout aussi insatisfaisante en ce qui concerne le traitement. C'est ainsi, par exemple, que selon l'article 25 de la Loi, un enfant de moins de douze ans peut être incarcéré si la Cour décide que " le bien de cet enfant et l'intérêt de la société rendent cette incarcération nécessaire (S.R., c. 160, art. 25), ce qui reflète déjà en soi l'esprit de la législation. Il n'en reste pas moins que c'est en 1971 seulement qu'on a présenté des amendements à la Loi. À ce moment, le ministre chargé de la patronner en Chambre avait déclaré : « La Loi concernant les jeunes délinquants n'a subi aucune modification importante depuis l'année 1929, ce qui signifie que depuis longtemps elle a cessé de refléter certaines préoccupations particulières à notre époque. s

\section{B. LE PROJET DE LOI C-192}

\section{ET LE CONCEPT DE LA RESPONSABILITÉ}

Le projet de Loi C-192 était en principe le fruit d'une longue réflexion collective.

Tout d'abord, en 1961, soit dix ans plus tôt, le ministère de la Justice fédérale a créé le Comité chargé d'étudier le problème de la délinquance juvénile au Canada, et qui a déposé son rapport en 1966. Cela signifie que la période d'enquête et de réflexion a duré cinq ans. Ensuite, en janvier 1968, il y a eu une conférence fédérale-provinciale où le problème et les recommandations du Comité ont été longuement discutés.

Il en est ressorti un projet de Loi dont le principal souci semble être celui d'unifier l'application de la législation à travers l'ensemble du pays, et non pas d'assurer une plus grande protection aux jeunes trouvés coupables d'un délit. Rien d'étonnant dès lors que la Loi ait été finalement rejetée en Chambre et renvoyée à un comité parlementaire pour étude.

Depuis, on attend donc à nouveau...

Compte tenu de ce qui s'est passé jusqu'à présent, il nous semble important, toutefois, de refaire l'autopsie du projet de Loi C-192 puisque rien ne prouve qu'il ne sera présenté, cette fois-ci 
en troisième lecture, avec quelques autres changements plus ou moins mineurs.

\section{ACTES CRIMINELS ET INFRACTIONS}

Le Bill C-192 précisait tout d'abord la dichotomie qui existe entre les actes criminels et les infractions à une loi provinciale, ou à un règlement municipal, en limitant le nombre et la catégorie des comportements dans le cas desquels la Loi peut s'appliquer.

A l'article 2, paragr. m), il était stipulé en effet que : \& Infraction », désigne une infraction qualifiée telle par une loi du Parlement du Canada, ou par toute ordonnance rendue en application d'une telle loi, toute règle ou tout règlement administratif ou autre, établis en vertu d'une telle loi, ou un outrage au tribunal en matière criminelle qui n'est pas commis à l'audience même ». La définition de « jeune délinquant », telle qu'elle figure dans la loi actuelle (art. 1 paragr. 2) devait être remplacée, en somme, par celle de l'acte, ce qui constituait incontestablement un amendement qui s'imposait.

\section{L'ÂGE DE LA RESPONSABILITÉ}

En deuxième lieu le projet de la Loi reconnaissait de façon formelle que l'âge de la responsabilité doit se situer à dix ans, tout en consacrant l'état de fait en rapport avec la limite de 16 , 17 ou 18 ans jusqu'à laquelle s'applique l'excuse atténuante de la minorité dans les diverses provinces canadiennes. A cet égard il s'agissait des deux textes suivants :

* Enfant $>$ désigne, selon le cas, soit une personne apparemment ou effectivement âgée de moins de dix-sept ans, à laquelle s'applique une proclamation lancée en vertu de l'article 3 (art. 2 paragr. c).

* Adolescent > désigne un enfant apparemment ou effectivement âgé de dix ans ou plus et, lorsque le contexte l'exige comprend une personne qui, en vertu de l'article 29 (concernant l'aveu ou la preuve du délit) fait l'objet d'une conclusion spécifique portant qu'elle a commis une infraction, jusqu'à ce que cette personne atteigne l'âge de vingt et un ans (art. 2 paragr. a).

\section{LA LEGISLATION PROVINCIALE}

En troisième lieu enfin, le projet de la Loi consacrait la latitude dont disposent en fait les juges des enfants d'appliquer 
la législation provinciale de protection de l'enfance de préférence à la législation criminelle. C'est ainsi que selon l'article 30 :

Lorsqu'un juge conclut spécifiquement qu'un adolescent a commis une infraction, il doit examiner le rapport préalable à la disposition établie, le cas échéant, en vertu de l'article 35 et tout autre renseignement pertinent et important, après quoi, le juge peut prendre l'une des dispositions suivantes ou un certain nombre d'entre elles à la condition qu'elles soient compatibles (paragr. 1c.).

Lorsqu'à son avis la preuve révèle qu'il serait au mieux des intérêts de l'adolescent de procéder en vertu d'une loi provinciale ayant pour but la protection ou le bien des enfants ou des adolescents, il peut libérer l'adolescent; pour que l'affaire puisse être poursuivie en vertu de cette loi (i), ou pour procéder lui-même en vertu de cette loi s'il a compétence en vertu de celle-ci (i).

\section{LA RESPONSABILITÉ DES PARENTS}

Compte tenu des insuffisances de la Loi actuelle, il était loisible de prétendre que les amendements cités plus haut représentaient un pas en avant, mais ils n'en demeurent pas moins indéfendables en tant que tels. Tout d'abord, en ce qui a trait à lâge de responsabilité, soit dix ans, il a été relevé dans la plupart des pays occidentaux à treize, à quatorze, et en Suède, à quinze ans. Présenter en 1974 et même en 1971, une loi qui le situe à dix ans, est en somme un anachronisme. C'est là, en outre, une façon de consacrer la confusion qui existe entre tout le problème de protection de l'enfance malheureuse, ou en danger, et celui du concept même de la «délinquance juvénile. » On le sait; toutes les études, enquêtes et recherches prouvent que les jeunes qui passent devant les cours pour mineurs sont, pour la grande majorité, des enfants dont la famille n'a pas pu ou su assumer ses responsabilités. Qu'on attribue ce phénomène à la pauvreté, à la dissolution des liens matrimoniaux, à l'alcoolisme, ou à la bêtise, importe peu. Le fait demeure que les juges rencontrent rarement dans la salle d'audience des enfants élevés dans des conditions qu'on peut définir comme favorables au développement harmonieux d'un être jeune. Souvent on pourrait parler là de la « délinquance parentale », mais infiniment plus rarement de la «délinquance juvénile ».

Or le problème de la «délinquance parentale » n'a pas été résolu, à notre connaissance, dans aucun pays du monde, de 
façon qui pourrait être considérée comme socialement satisfaisante. Dans le droit français existe la déchéance des droits paternels qui est absente dans notre législation, mais la déchéance ne peut être prononcée de toute façon qu'à postériori. En d'autres termes quand les parents perdent leurs droits à l'éducation de leurs enfants, il est déjà trop tard pour éviter à l'enfant les dommages qui ont été causés à sa personnalité. C'est là une réalité qu'aucune loi ne peut compenser, mais la question demeure entière : on vote une loi pour qui et pourquoi ?

Si on considère que la loi est faite pour punir les agissements d'un individu qui rejette les normes communément adoptées par la société, elle ne peut s'appliquer à un enfant de dix ans parce qu'il ne les connaît même pas, compte tenu de son âge. La fameuse règle : « Nul n'est sensé ignorer la loi », ne saurait être invoquée ici.

$\mathrm{Si}$, par contre, on reconnaît à la société le droit et l'obligation de se protéger contre les agissements qui menacent sa sécurité et son ordre, on voit mal la nécessité de le faire quand il s'agit d'un enfant de dix ans...

Il serait plus logique, si en pratique cela pouvait être possible, d'éliminer la «nuisance » des parents qui élèvent des enfants " incontrôlables », "vicieux», etc. et traiter les dits enfants de façon à ce qu'ils puissent se guérir de leurs mauvais penchants.

Le nœud du problème de traitement de l'enfant, dans le sens le plus classique de ce terme, demeure celui de l'accord des parents, d'une part et des disponibilités de la communauté, d'autre part. C'est donc là une dimension surtout sociale, tandis que l'intervention judiciaire se limite à assumer le rôle de l'arbitre entre les services sociaux et la famille quand apparaît un conflit relatif à la façon suivant laquelle l'enfant doit être éduqué et élevé.

On pourrait donc espérer que la nouvelle loi, tant attendue, reconnaisse l'irresponsabilité totale des enfants, au moins jusqu'à l'âge de quatorze ou quinze ans, limite au-delà de laquelle le jeune peut présenter un degré de dangerosité qu'une société donnée a droit de considérer comme inacceptable. Au lieu de juger les agissements de l'enfant, le magistrat serait appelé dès lors à statuer sur la demande des parents désireux de renverser la décision d'un service social préposé à assurer la protection de l'enfance. 
Or, le projet de Loi C-192 consacre uniquement une situation de fait en autorisant le juge à traiter l'enfant en vertu de la loi provinciale de protection, ce qui revient à imposer aux magistrats une responsabilité d'autant plus lourde que les cours pour mineurs sont les seules, en pratique, dont les sentences ne sont pas exécutoires, compte tenu de l'autonomie des institutions et des services de surveillance et de placement. Pis encore, étant donné la surcharge des agents de probation, comme des écoles de protection, le juge a beaucoup plus de chances de soustraire un jeune à l'influence préjudiciable de sa famille, en l'entendant en vertu de la Loi des Jeunes Délinquants, plutôt que d'une loi provinciale de protection.

Dès lors, demander aux magistrats de décider « en leur âme et conscience > est une hypocrisie tout à fait inacceptable de la part du législateur. L'objectif visé n'est pas, en somme, celui de protéger l'enfance et la jeunesse, mais d'en décliner la responsabilité au profit de celle des provinces.

\section{LA DÉFINITION DES DÉLITS}

La même remarque s'applique, également, à la dichotomie entre les actes définis comme criminels par le Code canadien et les législations ou règlements provinciaux et municipaux.

En effet, selon la législation proposée, la définition du délit ne concerne que les infractions désignées comme telles par une loi du Parlement du Canada, ce qui n'est pas le cas, par exemple, pour les infractions relatives aux lois de circulation qui relèvent des provinces, ou des municipalités. Or, c'est justement cette catégorie d'infractions qui, numériquement, est importante quand il s'agit des jeunes. Les garçons et les filles de moins de quinze ans vont donc continuer à défiler devant les cours, qui pour une bicyclette sans lumière, qui pour l'absence d'une plaque, bien qu'on sache fort bien que ce sont là des responsabilités qui incombent aux adultes, parents, ou tuteurs.

La même constatation s'applique en ce qui a trait à la consommation des boissons alcooliques.

La question qui se pose globalement est la suivante : un mineur de moins de quinze ans, doit-il être considéré comme responsable quand il est trouvé dans une taverne, ou est-ce qu'on doit convoquer à la cour les parents pour se rendre compte de 
la signification réelle du comportement de l'enfant, et imposer une lourde amende au tavernier?

Il ne s'agit pas en somme de savoir si la loi fédérale est plus ou moins répressive, mais si elle apporte une philosophie plus juste en ce qui a trait à tout le concept de la responsabilité de l'enfance. Faute de vouloir trancher et de statuer qu'aucun mineur de moins de quinze ans ne peut être jugé comme jeune délinquant, le législateur pourrait fort bien franchir une première étape en décidant, par exemple, que tous les enfants de moins de quinze ans doivent être traités uniquement selon les législations provinciales, ce qui reviendrait en fait au même.

\section{LE PROJET DE LOI C-192 ET LES DROITS DES MINEURS}

On a souvent prétendu, et à juste titre, que la justice des enfants est * paternaliste s. Le projet de loi a voulu, semble-t-il, rendre le cadre procédural plus précis et mieux délimité. En premier lieu la loi autorise le jeune qui a reconnu sa culpabilité de demander de recevoir sa sentence non pas dans la province où il a commis son délit, mais dans celle où il demeure. C'est là un amendement qui consacre un état de fait.

\section{L'INTERVENTION DU DÉFENSEUR LEGAL}

Au Québec la limite d'âge étant de dix-huit ans, tandis qu'en Ontario, de seize, les juges communiquent généralement entre eux afin de renvoyer notamment les québecois devant leurs cours. Actuellement, par ailleurs, un québecois peut toujours demander au juge d'une cour d'Ontario de suivre cette procédure, sachant qu'il a ainsi une chance, dans le cas de certains délits, d'éviter la prison. La situation inverse, par contre, ne s'applique pas et au Québec, ce sont paraît-il, surtout les ressortissants de l'état de Californie qui insistent pour recevoir leur sentence chez eux...

Le projet de loi prévoit néanmoins la possibilité également pour un jeune de demander de recevoir sa sentence non pas dans le district judiciaire où il a commis son délit, mais dans celui où il habite et c'est là un amendement lié à l'esprit même de l'article 26 (paragr. 2). Selon cet article la cour ne doit pas accepter d'un adolescent l'aveu d'une infraction qui lui est imputée dans une dénonciation s'il n'est pas assisté par un avocat, ou par ses 
parents, ou encore par un adulte, qui, de l'avis du juge, est capable de le conseiller. En effet, on peut présumer qu'un jeune aura de plus grandes possibilités de s'assurer le concours de sa famille quand il est jugé dans le district judiciaire où il est domicilié.

En ce qui a trait à la présence de l'avocat et au droit à la défense pleine et entière, comme c'est le cas de la procédure pour adultes, la situation demeure particulièrement complexe. D'une part, se pose là le problème on ne peut plus concret de la possibilité de disposer d'un défenseur à titre gratuit, et d'autre part, celui du rôle que ce défenseur devra assumer. Dans tous les cas, par exemple, où existe un conflit entre les parents et l'enfant, l'avocat devra-t-il défendre la position des parents ou celle de l'enfant? Quand, de l'avis des services sociaux et du juge, le placement s'impose dans l'intérêt de l'enfant, l'avocat devra-t-il plaider le retour au foyer familial ?

Il est évident, néanmoins, qu'à l'époque actuelle, les jeunes devraient pouvoir être informés de leurs droits et avoir un défenseur quand ils sont accusés de délits graves. On peut se demander dès lors si les cours pour mineurs ne doivent pas disposer d'un certain nombre de défenseurs légaux chargés d'assumer ce rôle, tout en laissant à l'enfant la possibilité de demander, s'il le désire, un autre avocat.

\section{LE DROIT D'APPEL}

Le droit à la défense pleine et entière est lié également à celui d'interjeter appel. Or, la procédure qui existe à cet égard est complexe et coûteuse et il serait normal qu'on la rende gratuite et qu'on la simplifie afin que les adolescents puissent profiter de ce recours. On peut aussi se poser la question en ce qui a trait à la création d'un tribunal d'appel à l'intérieur des structures de la cour pour enfants. Entendu par un juge seul, le jeune pourrait appeler de sa décision et être entendu par un tribunal de trois juges ayant le pouvoir de maintenir ou de renverser la décision qui a été prise à son égard. Un tel tribunal d'appel pourrait fort bien se réunir à intervalles réguliers de façon à ce que les procédures soient aussi souples et aussi accessibles que possible. À cet égard encore, le projet de Loi C-192 amorce une nouvelle tendance, mais ne va pas au-delà des mesures conservatrices qu'on ne peut considérer comme satisfaisantes. 


\section{LE MANDAT ET LA SOMMATION DE COMPARAÎTRE}

En ce qui a trait à la protection des droits des jeunes, la Loi C-192 prévoit, en outre, deux autres changements par rapport à ce qui existe actuellement. C'est ainsi qu'elle stipule que le juge, le juge de paix, ou le greffier, ne devront pas décerner de mandat à moins qu'il ne croie, en se fondant sur des motifs raisonnables et probables, qu'il est nécessaire, dans l'intérêt public, de décerner un mandat plutôt qu'une sommation. Parallèlement, la sommation, comme le mandat, devront comporter l'énoncé de l'infraction imputée et spécifier que l'adolescent auquel ils sont adressés a le droit d'être représenté par un avocat de son choix.

\section{L'ARTICLE 23 ET LE TRAITEMENT SOCIAL}

L'article 23 autorise, par ailleurs, le juge de désigner une personne pour s'entretenir avec le dépositaire d'une dénonciation en vue du règlement de la cause sans audition de l'adolescent.

Cette procédure ne peut être utilisée cependant qu'à condition que le procureur général de la province ne s'y objecte. Elle prévoit la préparation et la remise par la personne désignée d'un rapport écrit dans un délai ne dépassant pas deux mois et un tel règlement officieux interdit toute nouvelle procédure.

Les deux réformes sont incontestablement fondamentales puisque la première vise à limiter le nombre d'arrestations et informer mieux les jeunes de la nature de l'infraction qui leur est reprochée, comme du droit d'être défendu par un avocat, tandis que la deuxième permet à certains d'éviter la comparution à la cour. Comme il s'agit cependant d'un règlement officieux qui interdit toute nouvelle procédure, il est loisible de prétendre qu'il peut donner lieu à des abus, d'autant plus graves que le principal intéressé, c'est-à-dire l'adolescent, est privé, dès lors, de toute possibilité de recours. Par ailleurs, la procédure de ce type peut favoriser les ententes entre les services sociaux et le juge, mais ce dernier va se prononcer sur la base des documents sans voir l'enfant et sans pouvoir se faire une opinion personnelle en ce qui le concerne. Rien ne nous garantit, en somme, que l'intervention judiciaire sera pleinement valable et ne viendra pas consacrer uniquement la décision prise par les services sociaux, même si elle va à l'encontre des choix de l'adolescent, ce qui signifie qu'elle risque de devenir ainsi inopérante en termes de traitement. 
L'article 23 du projet de Loi est d'autant plus important qu'il constitue, en quelque sorte, l'exemple des contradictions qui existent en ce qui a trait à l'esprit de l'ensemble de la législation proposée. On a l'impression, en effet, que la Loi C-192 a été pensée non pas en fonction des intérêts des adolescents et des enfants, mais surtout de façon à unifier l'application de la loi à travers l'ensemble du pays.

\section{LES PROBLÈMES DE L'APPLICATION DES NOUVELLES PROCÉDURES}

C'est ainsi qu'on a voulu, semble-t-il, assurer une procédure plus précise et mieux uniformisée, mais sans se préoccuper des impératifs de son application. En dernier lieu, on a consacré l'état de fait, tout en évitant de situer clairement le rôle des services sociaux par rapport à ceux qui sont judiciaires et qui, en principe, continuent à détenir toute l'autorité. Dans cette optique, l'article 23 représente une exception où il est évident qu'on a voulu tenir compte à la fois des effets stigmatisants de la comparution en cour, de l'efficacité des modes de procéder autres que celui-ci et d'une autonomie, on ne peut plus partielle d'ailleurs, des services sociaux qui ne sont même pas définis dans la loi.

L'article de la loi autorise, en effet, le juge à désigner une personne pour s'entretenir avec le dépositaire d'une dénonciation en vue du règlement de la cause, sans audition de l'adolescent. On peut présumer donc que le juge autoriserait éventuellement les services sociaux à 's'entretenir avec la police afin de donner suite à la plainte. La personne désignée est tenue cependant de préparer un rapport écrit dans un délai ne dépassant pas deux mois et de le remettre au juge qui devra alors statuer.

On reconnaît là l'idée que le service social peut faire enquête sans intervention du pouvoir judiciaire et que ce dernier ne sera appelé dès lors qu'à trancher la question au niveau décisionnel. Il aurait suffi, en somme, d'élargir et de compléter l'article 23, singulièrement en ce qui a trait aux adolescents et aux enfants de moins de quinze ans, pour changer complètement toute la philosophie du traitement des mineurs au profit de la philosophie de protection, comparativement à celle qui découle du concept même de la délinquance. Il conviendrait cependant de donner à l'enfant le droit d'appel, au risque de conserver l'approche paternaliste et arbitraire. 
La dernière remarque qui s'impose a trait à tout l'aspect de la protection de l'enfant à travers une meilleure information du juge en ce qui concerne la situation réelle dans laquelle le jeune se trouve.

C'est ainsi que, d'une part, la loi stipule que quand une sommation est adressée à un adolescent, ou quand il est arrêté, les parents doivent être avisés de leur obligation de comparaître avec lui et s'ils ne s'y rendent pas, ils sont passibles d'outrage au Tribunal. Le greffier doit prévenir également, dans un délai raisonnable avant l'audition, un agent de probation attaché au tribunal, afin qu'il puisse y assister. Enfin, dans tous les cas où la cause d'un adolescent, âgé de plus de quatorze ans, doit être renvoyée devant un tribunal pénal ordinaire, le juge ne pourra rendre son ordonnance sans : \& faire faire sous sa surveillance une enquête sur les antécédents de l'adolescent et sur les circonstances de l'infraction imputée et, à cette fin, il pourra ordonner tout examen médical, psychologique ou psychiatrique, ou toute enquête sociale qu'il croit souhaitable s (art. 24 , paragr. 1 et 2 ).

Théoriquement, la loi ne vient que consacrer un état de fait puisqu'il est désormais d'usage de demander la présence de l'agent de probation à l'audience, de lui faire rencontrer l'enfant avant et de le charger par la suite de préparer un rapport pour éclairer le juge. Il n'en reste pas moins que sur le plan pratique, on peut considérer que cette mesure, aussi valable puisse-t-elle être, ne fait que prolonger le paternalisme de notre justice à l'égard des enfants.

En effet, on décide, d'une part, que le jeune doit avoir accès à une défense pleine et entière, mais d'autre part, on ne lui demande pas son avis, ni à son défenseur légal non plus, pour faire enquête dans sa famille et dans son entourage. Il est assez significatif à cet égard que, dans certaines provinces, les agents de probation des cours pour mineurs ne veulent plus faire enquête et préparer des rapports pré-sentenciels en affirmant que c'est là le travail de la police et que leur rôle devrait se limiter à la surveillance et à l'aide aux mineurs, placés sous leur responsabilité à la suite d'une décision du juge.

Si l'enfant est défendu par un avocat, on peut présumer que ce dernier utilisera dans sa plaidoirie tous les arguments concernant le passé du jeune, susceptibles de justifier directement, ou indirectement, son acte. Plus encore, si on admet qu'un enfant 
de plus de dix ans et de moins de quatorze a déjà assez de discernement pour reconnaître ou nier sa culpabilité, demander un avocat, demander qu'on entende sa cause en appel, etc., pourquoi ne lui donne-t-on pas en même temps le droit de demander au juge de l'isoler de sa famille de façon à ce qu'il ne soit plus obligé de la subir?

En d'autres termes pourquoi on ne l'autorise pas aussi à choisir, parmi les mesures que le juge a à sa disposition et qui s'appliquent dans son cas, celle qui paraît la plus conforme à ses besoins?

Pourquoi ne lui demande-t-on pas s'il accepte qu'on fasse une enquête dans son milieu, surtout quand il s'agit de délits mineurs, dans le cas desquels une telle enquête ne serait pas ordonnée s'il était adulte?

La difficulté de répondre à ces questions nous ramène une fois de plus à la constatation de base en ce qui a trait à l'ensemble de la Loi C-192. Certains articles de cette loi sont pensés de façon à protéger effectivement les droits d'un adolescent. En termes de jeunes ayant 15 ans et plus, il s'agit de mesures logiques et applicables, mais si on envisage les mêmes mesures à l'égard d'un enfant de moins de quatorze ans, elles sont parfaitement théoriques. Ce ne sont pas les droits de l'enfance que la justice protège là, mais bien plus ceux des parents, même s'ils sont responsables ou indignes, en paralysant tout aussi bien l'action des services sociaux que le pouvoir décisionnel du juge.

\section{LA RESPONSABILITÉ COLLECTIVE ET LES DROITS DE L'ENFANCE ET DE LA JEUNESSE}

Parallèlement, la Loi C-192 ne fait qu'atténuer la responsabilité des parents et il $\mathrm{y}$ a lieu de se demander si l'article 48 (paragr. 3a et paragr. 6a et $6 \mathrm{~b}$ ) ne doit pas être révisé de façon à autoriser les juges des enfants à prononcer des peines plus sévères à l'égard des parents dont, par exemple, la déchéance des droits. On sait, en effet, que dans les cas de placement en foyer de substitution, l'intervention des parents, et notamment leur droit de visite, rendent souvent inopérante cette mesure et cela au préjudice de l'enfant. Il est curieux également qu'à l'article 60 (paragr. 1 et 4c) la loi ne précise pas que les représentants de la presse doivent être autorisés à assister à l'audition de l'adulte, 
coupable de cruauté, ou de négligence à l'égard d'un mineur et de publier un compte rendu.

Il nous semble que, pour sensibiliser l'opinion publique, il est beaucoup plus important de l'informer des conditions dans lesquelles l'enfant a été obligé de vivre que de l'importance de l'acte délinquant qu'il avait commis et qui n'a été, fort souvent, que la résultante de la formation première reçue au foyer familial.

Dans son ensemble, la Loi, qui doit remplacer la Loi actuelle des jeunes délinquants, repose, en fait, tout le problème de la responsabilité collective à l'égard de l'enfance.

Comme nous l'avons vu au début de cet article, la Loi des Jeunes Délinquants, telle qu'elle existe encore actuellement, reffète l'esprit du début du siècle et consacre le principe de l'autorité absolue. La société décline toute responsabilité en ce qui a trait aux causes des agissements délinquants des enfants et des adolescents et se reconnaît uniquement l'obligation de les empêcher de nuire, ou même de déranger tout simplement, l'ordre public. Par opposition à son attitude à l'égard des adultes, elle se propose cependant non pas de punir, mais de «traiter $\gg$, ce qui souvent, en pratique, revient au même, malgré toutes les bonnes intentions.

Après quarante ans et une dizaine d'années de réflexion plus ou moins collective, on vient de se décider d'amender la Loi de 1929. On dit, et on le répète, à travers les rapports des commissions d'enquête, comme à travers les mémoires des divers organismes, que la nouvelle loi doit être conforme à l'esprit de notre équipe. On convient notamment que la société doit se reconnaître responsable de la détérioration des êtres jeunes qui troublent son ordre, respecter leurs droits individuels et les traiter en vue de leur resocialisation. Et tous ces termes ont en pratique une signification précise qu'on devrait retrouver dans l'esprit de la nouvelle loi.

\section{LA RESPONSABILITÉ COLLECTIVE ET LES DROITS DE L'ENFANCE}

En premier lieu, il faut se demander si le droit fondamental de l'enfance, face à la collectivité, n'est pas celui d'une irresponsabilité totale. Cela signifie que le juge des enfants devrait être leur défenseur face aux parents et face à la société. Jusqu'à l'âge de quatorze ans, tout du moins, il s'agit donc de protection et le 
terme même de « délinquance » paraît inapproprié puisque le concept même de culpabilité, ou de non-culpabilité, implique la connaissance préalable de règles morales ou autres, que les concernés n'ont généralement pas.

La loi fédérale, la loi qui figure dans le Code criminel, ne devrait donc pas se préoccuper des \& enfants \$, mais uniquement des adolescents ,, sinon on risque toujours de reproduire une sorte de parodie de la justice, en imposant aux juges une tâche qu'ils ne peuvent assumer. Compte tenu, toutefois, du fait qu'à l'égard des enfants la responsabilité de la collectivité demeure entière, il serait plausible que la loi fédérale se contente de prévoir les peines pour les parents et adultes cruels, délinquants, vicieux ou indifférents, et qu'elle prévoie l'obligation pour les autorités concernées de remplir pleinement les devoirs qui leur incombent.

Ả ce sujet, on comprendrait que la Loi impose à toute cour pour mineurs des obligations, dont par exemple, celle de disposer de cliniques d'observation et de traitement, ou encore d'un certain nombre d'agents de probation, calculé selon l'importance de la population âgée de moins de quinze ans de chaque district judiciaire.

En ce qui a trait aux procédures définies par la Loi, elles devraient aller au-delà des limites strictement judiciaires et se préoccuper de l'ensemble du problème de la protection.

En premier lieu, la loi devrait stipuler qu'aucun enfant de moins de quinze ans ne puisse être interrogé par la police autrement qu'en présence d'un travailleur social chargé de l'informer de son droit de ne pas répondre et de l'assister.

En deuxième lieu, la Loi devrait préciser que le juge interviendrait uniquement pour décider, de concert avec le travailleur social et les services sociaux dont il dépend, de la mesure à prendre pour assurer à l'enfant le meilleur milieu possible pour sa formation ultérieure.

En troisième lieu, dans tous les cas de placement dans un foyer de substitution ou dans une école de protection, l'enfant devrait avoir non seulement le droit, mais aussi la possibilité réelle et entière de communiquer avec les services sociaux et avec le juge.

On sait qu'actuellement les enfants subissent dans certains foyers de substitution des traitements aussi injustes et aussi préju- 
diciables à leur développement que dans leurs propres foyers d'origine, mais qu'ils n'osent pas, ou ne peuvent pas le dire à l'agent de probation parce qu'il est débordé, surchargé et incapable d'établir et de maintenir un climat de confiance entre lui et l'enfant. Tout l'objectif de traitement de ce type est dès lors faussé, sinon contraire à l'intérêt de l'enfant.

En quatrième lieu, enfin, la Loi pourrait fort bien imposer à la collectivité l'obligation formelle de disposer d'un certain nombre de centres de réception ou écoles, destinés aux enfants ayant besoin de protection; nombre calculé selon l'importance de la population de cette catégorie dans chaque district judiciaire.

On sait qu'actuellement les juges des enfants ont beau décider que l'enfant doit être placé, ou tout simplement hospitalisé, et que cela n'a aucune signification pratique, parce que la collectivité ne dispose pas de services adéquats et suffisamment bien organisés pour respecter la décision judiciaire.

Jusqu'à présent seules les prisons sont obligées d'accepter tout le monde; dans les hôpitaux, on peut attendre plus de six mois avant qu'un enfant référé par la clinique d'une cour puisse être reçu et traité...

En somme, la loi devrait préciser surtout les obligations de la collectivité à l'égard de l'enfance, ou encore, si le législateur est désireux de respecter l'autonomie provinciale, décréter qu'il incombe aux autorités des provinces d'élaborer des législations de ce type, compte tenu d'un cadre général, défini comme étant un minimum.

\section{LA RESPONSABILITE COLLECTIVE ET LES DROITS DES ADOLESCENTS}

Pour la deuxième catégorie des mineurs, soit les « adolescents > âgés de quatorze, ou de quinze ans, à dix-huit ans, la Loi devrait prévoir par contre une reconnaissance des droits spécifiques et plus importants que ce n'est le cas pour les adultes. En effet, autant il est normal que la société veuille se protéger contre les dangers de victimisation, autant il est impérieux qu'elle reconnaisse, en ce qui concerne les adolescents, une part de responsabilité qui est, il va sans dire, beaucoup plus importante que ce n'est le cas quand il s'agit des adultes. Cela signifie que les adolescents ne devraient pas être interrogés par la police autrement qu'en présence d'un travailleur social, ou d'un avocat, qu'ils 
seraient assistés par un travailleur social, ou par un avocat lorsqu'ils comparaissent devant le juge et qu'ils pourraient profiter des procédures d'appel totalement gratuites.

Cela signifie aussi que les adolescents référés à une cour pour adultes et condamnés à la détention ne pourraient dans aucun cas être enfermés dans une prison.

C'est au niveau des limites d'âge que le législateur devrait d'ailleurs manifester son souci d'unification de l'application de la loi à travers l'ensemble du pays, en refusant notamment de reconnaître aux provinces le droit de limiter le concept de * jeunes délinquants » à ceux âgés de seize ou de dix-sept ans.

La majorité légale étant de dix-huit ans, on comprend mal pourquoi la loi ne pourrait pas consacrer cet état de choses dans le domaine de la délinquance juvénile en évitant ainsi à des milliers de jeunes le drame d'une incarcération. Qu'on affirme que dans les prisons les mineurs sont séparés des adultes, qu'on explique que le personnel y est entraîné de façon spéciale, le fait demeure : la prison est, comme le prouvent de nombreuses recherches, criminogène. Il est, par conséquent, vital de soustraire à ce type de milieu tous les jeunes qui, compte tenu de leur âge, ont, par définition, plus de chances de se forger un avenir positif que leurs aînés. Il ne s'agit pas là d'une attitude permissive de la part de la collectivité à l'égard des adolescents, mais plutôt d'un investissement qui, à long terme, est hautement rentable, étant donné la durée moyenne de la vie. Certes, il y aura toujours des adolescents qu'il faudra isoler de la collectivité pour les empêcher de nuire, mais « isolement » peut signifier séjour dans une «école spécialisée » et non pas incarcération.

La dichotomie entre les deux groupes, « enfants » et « adolescents > doit donc consister non pas à assimiler une partie du deuxième groupe aux adultes, mais plutôt à accentuer la ligne de démarcation, autant au niveau de la responsabilité en loi que du traitement différencié entre les deux groupes. Prétendre, par exemple, que puisque dans une famille normale, les plus jeunes suivent l'exemple des aînés et que c'est là une cohabitation profitable, donc dans une école de protection, les mêmes relations doivent être maintenues, est l'expression d'une hypocrisie sociale inqualifiable. Tous les travailleurs sociaux et tous les juges des enfants savent que les adolescents sont généralement des « récidivistes > et qu'avec l'âge, certaines caractéristiques s'accentuent 
souvent dans notre système actuel de traitement, au lieu de s'estomper. Il n'est que logique, dès lors, d'arrêter le processus avant et d'isoler le groupe des « enfants », même si certains sujets présentent déjà un degré de détérioration de personnalité comparable à celle de leurs aînés, voire même supérieure.

\section{LES DROITS DES JEUNES ET LES CONTRAINTES BUDGÉTAIRES}

Est-il possible de concevoir une loi fédérale susceptible de remplir tous les objectifs tels qu'énoncés plus haut?

Il nous est difficile de répondre de façon exhaustive à cette question. Faute de place dans le cadre de cet article, qu'il nous soit donc permis de mentionner uniquement que dans certains pays, dont la Suède, cela a été réalisé. Il ne s'agit pas, toutefois, de copier le modèle suédois qui reconnaît la séparation totale entre le traitement des enfants de moins de quinze ans, purement social et administratif et celui des adolescents de plus de quinze ans et jusqu'à dix-huit ans, qui est judiciaire.

En effet, tout en maintenant le cadre judiciaire, on peut établir la dichotomie entre les «enfants 》 et les «adolescents ». En fait, il suffirait d'élargir le cadre de la loi C-192, à son article 23, à tous les enfants de moins de quinze ans, pour que leur traitement devienne purement social, avec le droit d'appel à l'autorité du juge, chargé de trancher en dernier ressort les conflits entre les services sociaux, les parents et les enfants concernés.

Pour conclure, une remarque d'ordre général s'impose; le législateur a principalement pour mission de statuer sur la protection des droits de l'individu et sur ses responsabilités à l'égard de la société. Il n'est pas de son propos de tenir compte des considérations d'ordre économique découlant de l'évaluation des priorités des provinces. La Loi C-192, qui est une législation capitale, non seulement en ce qui concerne les droits de l'enfance et de la jeunesse, mais aussi toute la philosophie sociale de notre collectivité, est une loi de compromis. C'est un secret de polichinelle que les provinces considèrent qu'elles ne disposent pas de budgets suffisants pour pouvoir assurer l'équipement social indispensable pour le traitement des adolescents. Dès lors, la loi ne pouvait contenir de réformes majeures sous peine d'être inacceptable pour certains gouvernements provinciaux. 
Cela nous a valu une loi vidée de toute philosophie progressive dont l'unique avantage consiste à introduire une procédure, difficilement applicable, compte tenu des insuffisances de l'assistance judiciaire.

Or, autant sur le plan national qu'international, le Canada ne peut pas se permettre, à notre avis, d'adopter une nouvelle loi faisant figure à côté de législation anglaise, par exemple, pour ne citer que celle-là, de rétrograde. Il est vrai que quand les autorités provinciales ont demandé au gouvernement fédéral s'il prévoit la possibilité de subventionner les services destinés au traitement des mineurs délinquants, le Premier Ministre s'est contenté de lancer «on donnera quelque chose pour la délinquance juvénile... », sans préciser ni quand, ni quoi... Mais que les services de traitement et de prise en charge soient subventionnés dans le cadre de Perspective Jeunesse, ou de Horizons Nouveaux, cela n'a vraiment pas d'importance quand il est question d'élaborer et de présenter une nouvelle loi qui est appelée à demeurer l'expression de la philosophie sociale qui est la nôtre et qui doit, en principe, devancer toutes les réformes faites dans le domaine des législations criminelles s'appliquant aux adultes...

Il ne reste qu'à espérer, en somme, que le législateur parviendra, en révisant enfin, après les trois dernières années de réflexion la Loi C-192, à faire preuve d'une indépendance d'esprit, au moins tout aussi poussée que ce fut le cas récemment dans le prononcé de sentence de certains juges au Québec qui ont osé contredire et mécontenter les pouvoirs gouvernementaux parce qu'ils ont cru leur devoir de le faire... 